\title{
Binocular rivalry suppression interferes with phase adaptation
}

\author{
RANDOLPH BLAKE and MARY BRAVO \\ Northwestern University, Evanston, Illinois
}

\begin{abstract}
With extended viewing, a triangle-wave grating takes on an unstable, illusory appearance, such that at times it more nearly resembles a square-wave grating. Prior adaptation to a genuine squarewave grating temporarily reduces the incidence of this illusory percept, stabilizing the appearance of a triangle-wave grating. The present experiment measured the effect of adapting to a square-wave grating that was suppressed during binocular rivalry for a substantial portion of the adaptation period. Rivalry suppression reduced the effectiveness of the adaptation pattern, indicating that the neural site of adaptation follows the locus of suppression.
\end{abstract}

When the two eyes view different monocular stimuli, an observer rarely sees a complete composite of both stimuli. Instead, the two dissimilar views undergo alternating periods of dominance and suppression, the phenomenon known as binocular rivalry. At any given time, in other words, an observer is consciously aware of the input from only one eye; information from the other eye is lost, or suppressed at some point prior to consciousness.

We do not know exactly where within the visual pathways the processing of information from the suppressed eye is interrupted by binocular rivalry. Nonetheless, it is possible to use suppression as a milestone for charting the flow of visual information, based on the following reasoning: Perceptual processes that are hindered by binocular suppression must occur at points coincident with or subsequent to the site of suppression, whereas processes unaffected by suppression must occur prior to or parallel with binocular suppression. Following this line of reasoning, investigators have studied the effects of suppression on a number of visual processes, including stereopsis (e.g., Ogle \& Wakefield, 1967), pupillary light reflex (Barany \& Hallden, 1948), and visual aftereffects (Blake \& Fox, 1974; O'Shea \& Crassini, 1981; Wade \& Wenderoth, 1978).

The experiment described in this paper uses binocular suppression as a tool for determining the relative locus of phase adaptation. Several studies have demonstrated phase-specific adaptation effects, whereby some aftereffect of grating adaptation depends on the phase relationship of the adapting pattern's spatial frequency components (De Valois, 1977; Sansbury, Distelhorst, \& Moore, 1978; Stroymeyer, Lange, \& Ganz, 1973). One striking example of phase adaptation involves a recently dis-

This work was supported by a grant from the National Science Foundation (BNS 82-00850). We thank Karen Holopigian and Karin Boothroyd for their participation as observers. The authors' address is: Cresap Neuroscience Laboratory, Northwestern University, Evanston, IL 60201 . covered visual illusion, dubbed the square-wave illusion (Leguire, Blake, \& Sloane, 1981). The illusion occurs when one views a grating pattern with a triangle-wave luminance profile. After a few moments, such a grating begins to fluctuate in appearance: Sometimes it looks like a triangle-wave grating, whereas other times it more nearly resembles a square-wave grating. Adaptation to a true square-wave grating of the same spatial period temporarily reduces the incidence of the square-wave illusion (i.e., a subsequently viewed triangle-wave grating is seen predominantly as a triangle-wave grating). In contrast, adaptation to the fundamental alone has no effect on the illusion, nor does triangle-wave adaptation affect the appearance of a square-wave grating. (Readers may confirm these observations by following the instructions accompanying Figure 1.)

In the case of this illusion, what does adapting to a square-wave grating have to do with phase adaptation? It is noteworthy that a triangle-wave grating and a squarewave grating of equivalent fundamental frequency consist of the same harmonic components. In the square-wave grating, however, these components are in peaks-subtract phase, whereas for the triangle-wave grating they are in peaks-add phase. In the Fourier domain, in other words, phase represents the chief difference between square-wave and triangle-wave gratings. Couched in these terms, prior adaptation to peaks-subtract phase (i.e., square-wave grating) temporarily stabilizes the appearance of a subsequently viewed pattern whose components are in peaksadd phase (i.e., a triangle-wave grating). This is why the effect has been characterized as "phase adaptation." The purpose of the present experiment was to learn where in the sequence of visual processing phase adaptation occurs, relative to binocular suppression.

To answer this question, we measured the incidence of the square-wave illusion following several conditions of adaptation. In one condition, observers merely adapted to a homogeneous display, a condition providing baseline measures of the incidence of the illusion. In the second condition, observers monocularly adapted to a 

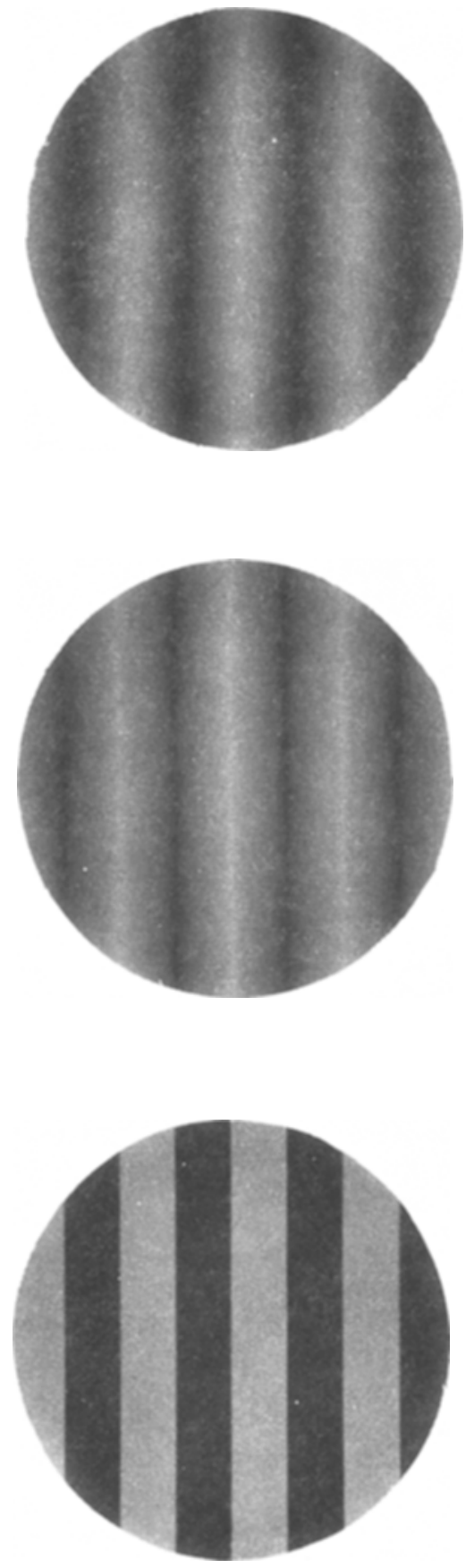

Figure 1. Using these three grating patterns, the reader may experience the square-wave illusion and may confirm that adaptation to a sine-wave grating (top) has no effect on the illusion, whereas adaptation to a square-wave grating (bottom) stabilizes the appearance of the triangle-wave grating (middle). Start by staring at the central light stripe in the triangle-wave grating. While holding your fixation as steady as possible, pay attention to the lightness of the regions just to the left and just to the right of this light stripe. The luminance profile falls off evenly on either side of the stripe, but after a few moments the apparent lightness appears to fluctuate. Once you have experienced the illusion, adapt for a minute or so to the sine-wave grating and then reinspect the triangle-wave grating. Now repeat this adaptation procedure, this time adapting to the square-wave grating. genuine square-wave grating; the nonadapted eye viewed an unpatterned display. In the third condition, observers monocularly adapted to a square-wave grating while the nonadapted eye viewed a "noise" display; in this condition, the adaptation grating was phenomenally suppressed for a substantial portion of the adaptation period, due to binocular rivalry. Our results show that suppression does interfere with the adaptation effects of the square-wave illusion, and thus indicate that this phase-adaptation process occurs after the site of binocular rivalry.

\section{METHOD}

Grating patterns were generated electronically on a pair of matched cathode-ray tubes (CRTs) that were viewed dichoptically via a mirror stereoscope (Blake \& Cormack, 1979). For each CRT display, the grating appeared within a circular area $1.66^{\circ}$ in diameter; the remainder of each eye's $5^{\circ} \times 7^{\circ}$ display consisted of black fusion lines superimposed on an evenly illuminated field (average luminance equaled $7 \mathrm{~cd} / \mathrm{m}^{2}$ ). The fundamental spatial frequency of all gratings, regardless of luminance profile, was 1.2 cycles $/ \mathrm{deg}$; thus, two complete cycles of the waveform appeared within the circular aperture. The contrast of the triangular-wave test grating was always $40 \%$; the contrast of the square-wave adaptation grating was $7 \%$, a value high enough to yield significant adaptation yet low enough to render that grating invisible during a substantial portion of the binocular rivalry viewing conditions.

In place of a vertical grating, one-dimensional horizontal noise could be presented within the circular aperture of one of the CRTs. This was accomplished by passing the output of an audio noise generator (Grason-Stadler, dc, $20 \mathrm{kHz}$ ) to the z-axis of the CRT. When one eye viewed this broadband noise and the other viewed a grating, vigorous binocular rivalry ensued. Because of the small size of the rival targets, the resulting suppression tended to be unitary, not piecemeal, thereby minimizing criterion problems for observers.

Three observers participated in this experiment. All had excellent visual acuity and stereopsis. Two of the three were naive about the hypothesis under test; the third was one of the authors.

\section{RESULTS}

\section{Growth of Adaptation with Time}

The logic of our experiment assumes that the strength of the aftereffect of adaptation to a real square-wave grating depends on the duration of adaptation: Brief adaptation should have less influence on the square-wave illusion than longer periods of adaptation. To assess the correctness of this assumption under the conditions of our experiment, we measured the incidence of the squarewave illusion following various durations of adaptation to a square-wave grating. The observer (R.B.) tracked the illusion during a $15-\mathrm{sec}$ test period by depressing a button when the triangle-wave grating resembled a squarewave grating. These test periods were preceded by a 1min adaptation period during which a square-wave grating was intermittently presented, thereby mimicking the phenomenal alternations of rivalry. The total duration of intermittent adaptation was varied from $0 \mathrm{sec}$ (adaptation to a blank display only) to $60 \mathrm{sec}$ (adaptation to a continuously visible grating) across blocks of trials. At least 10 min rest intervened between each condition.

Results confirmed that the incidence of the square-wave illusion is progressively reduced with increased cumula- 
tive exposure duration to a square-wave grating. Thirty seconds of adaptation, for instance, reduced the incidence of the square-wave illusion by approximately one-half. This finding merely sets the stage for our main experiment.

\section{Effects of Suppression on Square-Wave Adaptation}

In the main experiment, the following adaptation/test sequence was employed for each of four adaptation conditions. The observer initially adapted for $1 \mathrm{~min}$, then tracked the incidence of the square-wave illusion for $15 \mathrm{sec}$, readapted for $15 \mathrm{sec}$, tracked for $15 \mathrm{sec}$, and so on for a total of four $15-\mathrm{sec}$ test periods. The following four adaptation conditions were administered: (1) baseline condition.(B) - both eyes viewed an uncontoured display; (2) square-wave adaptation (SQ) - the left eye viewed a square-wave grating while the right eye viewed an uncontoured display; (3) noise adaptation (N)-the left eye viewed an uncontoured display while the right eye viewed one-dimensional noise; and (4) binocular rivalry (BR)the left eye viewed a square-wave grating while the right eye viewed one-dimensional noise. During the rivalry condition (BR), observers tracked the rivalry alternations between noise and square-wave adaptation grating by depressing a button whenever the grating was completely suppressed. Each condition was repeated three times, with the order of conditions randomized.

Results are summarized in Figure 2. The pattern of results was equivalent for all three observers, so we nor-

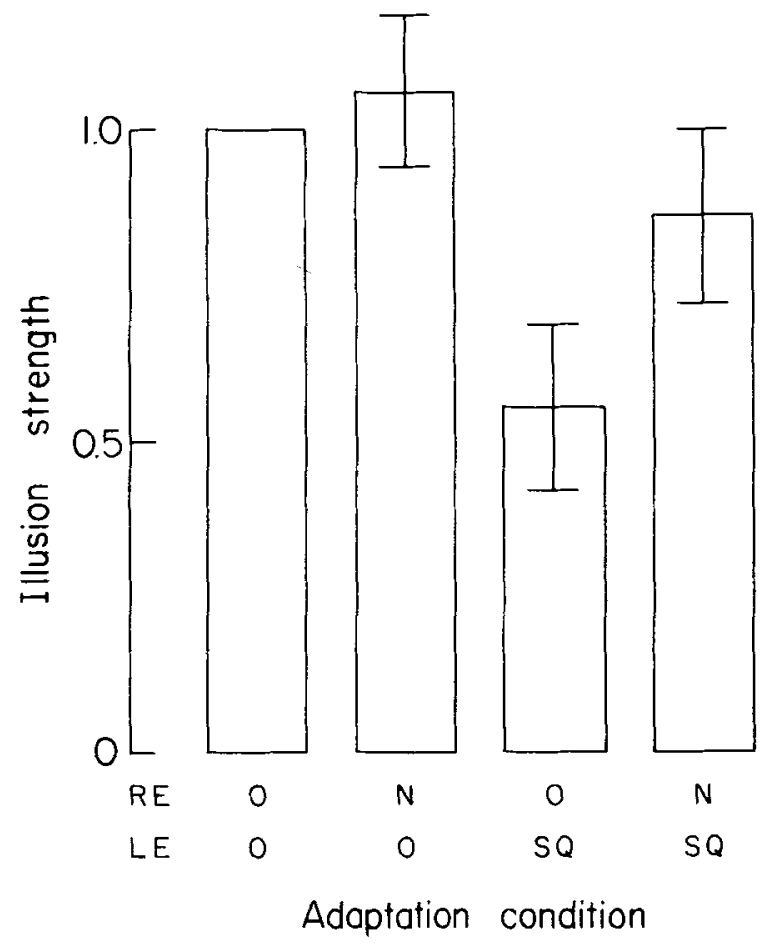

Figure 2. Illusion strength for different conditions of adaptation. From left to right, the four conditions are B, N, SQ, and BR. All data were normalized to those in Condition $B(O / O)$. malized each observer's data to the baseline condition and averaged the data across observers. Following adaptation to the blank display, the triangle-wave luminance profile appeared distorted about $35 \%$ of the total viewing time. Adaptation to noise alone ( $N$ ) had no effect on the incidence of the illusion, but adaptation to the square-wave alone reduced the incidence of the illusion significantly ( $p<.01$, Wilcoxon sign test). This outcome merely replicates earlier findings (Leguire, Blake, \& Sloane, 1982). Adaptation to the intermittently suppressed squarewave grating was less effective than adaptation to the continuously visible square-wave grating; the difference between conditions SQ and BR is statistically significant ( $p<.01$, Wilcoxon sign test).

Observers' tracking reports during BR adaptation indicate that the square-wave grating was phenomenally visible for an average total of $20 \mathrm{sec}$ during the 60 -sec adaptation period. Results from our initial pilot experiment showed that $20 \mathrm{sec}$ of adaptation distributed intermittently over $60 \mathrm{sec}$ yielded a threefold increase in the incidence of the illusion in comparison to Condition SQ, in which the adaptation grating was continuously visible. This mirrors very closely the change in illusion strength found in this main experiment (compare Conditions BR and SQ).

\section{DISCUSSION}

In terms of its effect on the square-wave illusion, intermittent phenomenal suppression of a square-wave adaptation grating produces the same result as actually presenting and removing that grating. Thus, whatever neural process is engaged during square-wave adaptation, binocular suppression effectively blocks the flow of information to that process. The site of square-wave adaptation, in other words, receives input from (and therefore must occur after) the locus of suppression. At the same time, we know from earlier work that suppression has no influence on the threshold elevation aftereffect produced by grating adaptation (Blake \& Fox, 1974), whereas grating adaptation does influence the duration of an eye's suppression during rivalry (Blake \& Overton, 1979). This pattern of results unambiguously implies that suppression occurs after spatial-frequency adaptation.

On putting these various pieces of evidence together, then, the following picture emerges. As an observer stares at a grating, that pattern adapts neurons at several distinct stages of visual processing. One stage occurs before, and provides input to, binocular suppression. Another stage occurs after, and receives input from, binocular suppression. Can we say anything about what role might be played by neurons at these two sites of adaptation? It is generally believed that neurons at the earlier site (where threshold elevation occurs) register information about spatial frequency and orientation (e.g., Blakemore \& Campbell, 1969). The second site of adaptation (which influences the appearance of a complex grating) is presumably involved in extracting phase information from 
patterns composed of multiple spatial frequencies (e.g., Atkinson \& Campbell, 1974; but see Rentschler \& Treutwein, 1985). Whether the specifics of this scenario are true or not, the differential effects of suppression on two aftereffects of grating adaptation clearly implicate several sites of adaptation with suppression intervening.

We should point out that the present results do not necessarily imply that the neural events underlying the illusion itself occur after binocular suppression. It is conceivable that neurons at an early stage of visual processing are responsible for the illusion, whereas those at a later stage (i.e., one following suppression) extract phase information and are subject to adaptation. Indeed, Georgeson and Turner (1984) believe that the illusion occurs retinally when eye movements superimpose an afterimage of a triangle-wave grating on the actual image of that same pattern. Alternatively, Leguire et al. $(1981,1982)$ attributed the illusion to instability among cortical phaseselective mechanisms operating in an opponent-process manner. The present results do not distinguish between these two theories concerning the origins of the trianglewave illusion. However, previous results show that the illusion can be seen when the triangle-wave target is briefly flashed or viewed as an afterimage (Leguire et al., 1981). These earlier observations go against the afterimage theory as a complete explanation of the illusion. Whatever causes this illusion, we now know that the site of adaptation where the incidence of this illusion is influenced occurs after binocular rivalry suppression.

\section{REFERENCES}

Atrinson, J., Campbell, F. W. (1974). The effect of phase on the perception of compound gratings. Vision Research, 14, 159-162.
Barany, E. H., \& Hallden, U. (1948). Phasic inhibition of the light reflex of the pupil during retinal rivalry. Journal of Neurophysiology, 11, 3-8.

Blake, R., \& CoRmack, R. (1979). On utrocular discrimination. Perception \& Psychophysics, 26, 53-68.

BLAKE, R., \& Fox, R. (1974). Adaptation to invisible gratings and the site of binocular rivalry suppression. Nature, 249, 488-490.

BLAKE, R., \& OVERTON, R. (1979). The site of binocular rivalry suppression. Perception, 8, 143-152.

Blakemore, C. , \& Campbell, F. W. (1969). On the existence of neurones in the human visual system selectively sensitive to the orientation and size of retinal images. Journal of Physiology, 203, 237-260.

De VALOIS, K. K. (1977). Independence of black and white: Phase specific adaptation. Vision Research, 17, 209-216.

Georgeson, M. A., \& Turner, R. S. E. (1984). Stability of phase recognition in complex spatial waveforms. Vision Research, 24, 851-858.

Leguire, L. E., Blake, R., \& Sloane, M. (1981). A novel visual illusion of bars made from triangles. Science, 212, 1172-1175.

Leguire, L. E., Blake, R., \& SlOANE, M. (1982). The square-wave illusion and phase anisotropy of the human visual system. Perception, 11, 547-556.

OGle, K. N., \& WaKefield, J. M. (1967). Stereoscopic depth and binocular rivalry. Vision Research, 7, 89-98.

O'Shea, R., \& Crassini, B. (1981). Interocular transfer of the motion after-effect is not reduced by binocular rivalry. Vision Research, 21, 801-804.

Rentschler, I., \& Treutwein, B. (1985). Loss of spatial phase relationships in extrafoveal vision. Nature, 313, 308-310.

Sansbury, R. V., Distelhorst, J., \& Moore, S. (1978). A phasespecific adaptation effect of the square-wave grating. Investigative Ophthalmology, 17, 442-448.

Stroymeyer, C. F., Lange, A. F., \& Ganz, L. (1973). Spatial frequency phase effects in human vision. Vision Research, 13, 2345-2360.

WADE, N. S., \& WENDEROTH, P. (1978). The influence of contour rivalry on the magnitude of the tilt after-effect. Vision Research, 18, 827-836.

(Manuscript received June 13, 1985; revision accepted for publication September 6, 1985.) 